\title{
Erratum to: Artificial Intelligence for Fashion Industry in the Big Data Era
}

\author{
Sébastien Thomassey and Xianyi Zeng
}

\section{Erratum to:}

S. Thomassey and X. Zeng (eds.), Artificial Intelligence for Fashion Industry in the Big Data Era, Springer Series in Fashion Business, https://doi.org/10.1007/978-981-13-0080-6

In the original version of the book, the following corrections have to be incorporated:

In chapter "AI-Based Fashion Sales Forecasting Methods in Big Data Era", the affiliation "Institute of Textiles and Clothing, The Hong Kong Polytechnic University, Hunghom, Kowloon, Hong Kong" of the co-author "Shuyun Ren" has to be changed as "Guangdong University of Technology, China".

In chapter "Blockchain-Based Secured Traceability System for Textile and Clothing Supply Chain", the incorrect affiliation "Independent consultant, Chennai, India" of the co-author "Ajay Sharma" has to be corrected so that it should read as "Independent Consultant, Roubaix, France".

\footnotetext{
The updated online version of these chapters can be found at https://doi.org/10.1007/978-981-13-0080-6_2 https://doi.org/10.1007/978-981-13-0080-6_10 https://doi.org/10.1007/978-981-13-0080-6 